\title{
Editorial: Mathematical Models for Intertemporal Choice
}

\author{
Salvador Cruz Rambaud * \\ Department of Economics and Business, University of Almeria, Almería, Spain
}

Keywords: intertemporal choice, anomalies, behavioral finance, mathematical model, decision making

\section{Editorial on the Research Topic}

\section{Mathematical Models for Intertemporal Choice}

In general, a wide variety of approaches are allowed in Mathematical Finance: one of them involves the implementation of mathematical models able to explain the complexity of real situations in Finance. In particular, intertemporal choice is gaining the attention of researchers because of its increasing application to other fields - such as psychology or health. Obviously, every model presents logical mistakes (or gaps), and intertemporal choice is not an exception. This was shown in the recent Research Topic labeled as "Intertemporal Choice and Its Anomalies".

The objective of this Research Topic was to describe intertemporal choices as mathematical models, as general as possible, with the aim to cover all possible situations and analyze the properties which can be useful for decision makers. Since most financial decisions include decision making over time, this Research Topic is aimed also at mathematical modeling of important anomalies such as Allais' paradox (violation of von Neumann and Morgenstern's independence axiom), mental accounting, and myopic loss aversion in behavioral finance (discovered by Nobel laureate Professor Richard H. Thaler and colleagues).

Also, in behavioral finance, Nobel laureate Professor Robert J. Schiller observed excessive volatility in comparison to streams of future dividends in the United States stock markets, which reflects inefficiency in the market and irrationality in people who trade stocks. Mathematical models which have implications for these anomalies in the markets are also within a scope of this collection. Furthermore, recent advances in neuroeconomics revealed the important roles of emotion in a decision over time and under uncertainty.

Jiwei Zhang, Jing Lu, Hang Du and Zhaoyuan Zhang introduce a new Gibbs slice sampling algorithm for estimating the four-parameter logistic model which has reached lot of interest in educational testing and psychological measurement. The sampling process was divided into two parts. The first part is the Gibbs algorithm, which was used to update the guessing and slipping parameters when non-informative uniform priors are employed for cases which are prototypical of educational and psychopathology items. The second part is the slice algorithm, which samples the 2PL IRT model from the truncated full conditional posterior distribution by using auxiliary variables.

Yang Lu, Jian Wang, Chenyang Li, Haoya Huang and Xintian Zhuang provide an extension of the paper by Hoelzl et al. (2011) and Cruz Rambaud et al. (2019) as they deal with the improving sequence effect in loans contexts. Traditionally, this anomaly of the intertemporal choice was reduced to choices between rising earnings and other increasing/decreasing sequences. In a beginning, previous studies have shown a consistent preference for falling sequences in the context of loan repayment plans. However, the results show that consumers follow a comparison-based decision making process rather than optimization when evaluating temporally reframed loan offerings. Individuals preferred the falling over the constant profile only if the interest rate was $10 \%$ and the loan profiles were described in a per-year form. 
On the other hand, Jiwei Zhang, Jing Lu, Jing Yang, Zhaoyuan Zhang, and Shanshan Sun introduce the so-called "Mixture Multiple Strategy-Deterministic, Inputs, Noisy and Gate (MMS-DINA) model in order to investigate individual differences in the choice of responses categories. The simulation indicates that the Markov chain Monte Carlo (MCMC) algorithm can be used to obtain accurate parameter estimates. Additionally, two Bayesian model assessment criterions are considered to evaluate the model fitting among DINA model, MS-DINA model and MMS-DINA model. Thus, it is shown that, when the data are generated from the simple singlestrategy DINA model, the MMS-DINA model fits the data better than the MS-DINA model.

Finally, Xuemei Xue, Jing Lu, and Jiwei Zhang introduce a multidimensional Rasch model for measuring learning and change (MRMLC) and its dichotomous and polytomous extensions is used in longitudinal study. Two simulation studies have been carried out to further illustrate the advantages of this item-weighted likelihood estimation method compared to the traditional Maximum a Posteriori (MAP) estimation method, Maximum Likelihood Estimation (MLE) method, Warm's Weighted Likelihood Estimation (WLE) method, and TypeWeighted maximum Likelihood Estimation (TWLE) method, resulting in a better recover examinees' true ability level for both complex longitudinal IRT models and unidimensional IRT models compared to the existing likelihood estimation methods.

\section{AUTHOR CONTRIBUTIONS}

The author confirms being the sole contributor of this work and has approved it for publication.

Conflict of Interest: The author declares that the research was conducted in the absence of any commercial or financial relationships that could be construed as a potential conflict of interest.

Publisher's Note: All claims expressed in this article are solely those of the authors and do not necessarily represent those of their affiliated organizations, or those of the publisher, the editors and the reviewers. Any product that may be evaluated in this article, or claim that may be made by its manufacturer, is not guaranteed or endorsed by the publisher.

Copyright (c) 2021 Cruz Rambaud. This is an open-access article distributed under the terms of the Creative Commons Attribution License (CC BY). The use, distribution or reproduction in other forums is permitted, provided the original author(s) and the copyright owner(s) are credited and that the original publication in this journal is cited, in accordance with accepted academic practice. No use, distribution or reproduction is permitted which does not comply with these terms. 